\title{
EDITORIAL
}

\section{The final act-a?}

Acta Neuropsychiatrica 2006: 18:233. (C) Blackwell Munksgaard 2006

This issue of Acta Neuropsychiatrica, the last in this particular format, publishes the scientific abstracts from the annual conference of the Australasian Society for Psychiatric Research (ASPR) that is taking place in Sydney this year. It begins with a scene-setting editorial from the Conference Convenor (1) that discusses the main theme of the meeting namely 'brainwaves'.

I would like to take this opportunity to thank the many reviewers and referees who have done a wonderful job this year, as without them, not a single paper could have been published. I am also grateful to the many contributors and subscribers that in addition to choosing to publish in this journal have made valuable suggestions as to how the journal should evolve. In conclusion, I have outlined below the significant changes that the journal will undergo in the New Year so as to enliven its content further.

The first issue of 2007 will be published in February. It will have a new cover depicting an image that has been chosen to convey the neural focus of the journal. The three-dimensional image, in addition to demonstrating connectedness of thoughts and ideas, is designed to imbue a sense of depth. This reflects the increased research interest in brain neurobiology and understanding its pathophysiology in the context of neuropsychiatric disorders. The journal will also benefit from several new sections that will feature particular themes.

The first of these sections called Pictures and Prose will showcase images and text relevant to neuropsychiatric illness. For instance, interesting pieces of art pertaining to mental illness or historical accounts of psychiatric disorders will be featured. Occasionally, a précis of a seminal paper or the original writings of a leading figure in psychiatry will be discussed. Another section called Insight Interventions will be written by leading researchers and will aim to provide a succinct review of new and emerging treatments so as to inform readers about novel therapeutic developments in neuropsychiatry. A third section termed Brain Bytes will provide a succinct review of technological advances and examine both hardware and software that are currently being used in neuropsychiatric research or have potential to be applied to the field. This section will be written in consultation with relevant experts but retain accessibility, so that readers can gain a practical understanding of experimental equipment. The new format will also include a regular piece on psychiatric statistics. This section, called Speaking Statistically, will form a series of articles that will aim to provide a working knowledge of statistics as relevant to readers and contributors of this journal. Finally, we will also be publishing book reviews and correspondence so as to facilitate useful and interesting scientific debate.

I look forward to your participation in the journal and thank those who have stimulated and supported the changes.

\section{Professor Gin S. Malhi \\ Head, Academic Discipline of Psychological Medicine Northern Clinical School University of Sydney}

\section{Reference}

1. Harris A. Editorial: Brainwaves. Acta Neuropsych. 2006;18:234-235. 